\title{
Impact of P2P Trading on Distributed Generation Curtailment in Constrained Distribution Networks
}

\author{
Mark Jenkins \\ Institute of Energy and Environment \\ University of Strathclyde \\ Glasgow, Scotland \\ mark.jenkins@strath.ac.uk
}

\author{
Ivana Kockar \\ Institute of Energy and Environment \\ University of Strathclyde \\ Glasgow, Scotland \\ ivana.kockar@strath.ac.uk
}

\begin{abstract}
The increasing uptake of Distributed Energy Resources and advancement in blockchain technology has led to the growth in interest in the peer-to-peer (P2P) based energy trading. However, there has been a lack of consideration how these trades may affect the network operation, in particular in networks that apply Active Network Management using Last-inFirst-out (LIFO) access rules, such as in the UK. This paper presents a novel application of Optimal Power Flow that manages the P2P trading whilst optimising legacy DGs under a LIFO access agreement. Analysis show that under such arrangements, and in combination with autonomous P2P trading, lower priority DGs are vulnerable to excessive curtailment levels.
\end{abstract}

Index Terms-- Peer-to-Peer trading, Bilateral Contracts, Curtailment, Distributed energy resources, Optimal Power Flow

\section{INTRODUCTION}

There is a significant increasing number of distributed energy resources (DERs) such as renewable energy sources, storage and domestic 'smart' appliances [1] becoming integrated into the power system. These technologies are having profound impacts on the way the power system is operated, particularly on the lower voltage distribution networks. As a result, Distribution Network Operators (DNOs) are modifying their traditional passive operating philosophy and adopting a more active role. Moreover, as penetration levels of these stochastic and intermittent DERs increase, the challenges become more prominent and complex. Inadequate management of DER integration can lead to both thermal and voltage violations. These can cause significant underutilisation of network capacity for future (and current) renewable generation [2], as well as blocking further connections of renewable resources.

Distributed Generation (DG) capacity was, in the UK, initially granted on a case-by-case basis through a so-called 'fit and forget' approach, where the DG was granted a firm allocation of capacity based on conservative network planning. However, low voltage network capacity became more limited, and, as a result, DNOs struggled to facilitate DGs with firm connections [2]. This posed a significant problem to DNOs who searched for innovative solutions to enable additional DG connections while avoiding costly network reinforcements.
The main approach tested in the UK, which has since become the Business as Usual (BaU), is Active Network Management (ANM). As described in [3], ANM includes a monitoring and control system that, in real time, enables management of DGs' outputs in the case where network limitations are reached.

For DGs integrated into ANM schemes, it is common to have a non-firm arrangement that is subject to curtailment and managed in accordance with a prearranged Principles of Access (PoA) agreement [4] and [5]. The most commonly used and easily implemented PoA for ANM schemes is the Last in First Out (LIFO) arrangement, under which the last DG to connect to the ANM scheme is the first to be curtailed, with initial connections having priority over network capacity. This arrangement has been embedded for a long time, under which many DGs have made investment decisions and to unbundling/changing this arrangement would be difficult process.

Furthermore, recent developments have seen a trend towards decentralised energy trading at the distribution level. This has been investigated both in research work and through a number of pilot projects [6]. For example, the use of Peer-toPeer (P2P) energy transactions in local markets to facilitate energy exchanges between small-scale energy producers and consumers has been investigated in [7-10]. In P2P electricity trading, 'peers' have the ability to directly and autonomously negotiate energy exchanges and prices with any other on the network, regardless of magnitude or location. However, debates on the specific coordination, architecture and management of this trading structure are still ongoing.

The authors in [11] have identified three prominent market platform designs that may influence the development in decentralised energy trading 1) Fully Peer-to-Peer Market, 2) Community-based Market and 3) Hybrid P2P market. The distinguishing components between the three deigns are the topology of allowable trades and the degree of which there is hierarchical oversight/control. The work presented in [12-13] are examples of Fully Peer-to-Peer Markets, that present market structures based on a bilateral economic dispatch formulation that allows for individual consumer preferences. An Example of a Community-based Market is presented in 
[14], which illustrates P2P trading within a $\mu$-grid where an energy sharing structure is proposed that integrates the peers into an energy sharing zone. In this zone, the peers are equal and in agreement on a unified price with intention that a unified $\mu$-grid is overall more economically beneficial (when compared to using a structure of individual self-interested participants). Lastly, [15] and [16] have proposed work that uses a Hybrid P2P market approach. In [15] a higher level of control is introduced that expands on the work from [14], where the $\mu$-grid itself acts as one 'peer'. Trading between the $\mu$-grid peers is detailed alongside a localized fully P2P market within the $\mu$-grid itself. In addition, [16] expands work from [15], with trading between peers (on the same network) solved through a relaxed form of Optimal Power Flow (OPF).

In practice, participants in $\mathrm{P} 2 \mathrm{P}$ markets will have restrictions imposed by network limits as well as technical constraints, and the absence of such consideration may lead to operational issues. Despite this, only a few studies have assessed the impact that fully $\mathrm{P} 2 \mathrm{P}$ trading mechanisms may have on the network [17, 18, 19]. In [18], unique network fees were imposed on certain transactions depending on the location and distance of the negotiated trade. These fees were informed through electrical distance methods such as Power Transfer Distance (PTD) and analysed using a DC power flow. A novel methodology based on a linearized power flow was presented in [19], which used partial derivatives of the Jacobian matrix to extract voltage and power flow sensitivities that are then used in an iterative process to solve the power flow.

It is the aim of this paper to illustrate the effects that P2P trading can have on the network operation, particularly when considering legacy DGs under a PoA agreement, i.e. LIFO. In order to do that, a full representation of network constraints in an AC OPF solution is required. Extending the work of [20], we introduced $\mathrm{P} 2 \mathrm{P}$ trading that, similarly to arrangements at the transmission level [21], are allowed without conventional intermediaries. We analyse the impact such trading can have on the levels of curtailment under various LIFO arrangements and in doing so demonstrate the need for further understanding when considering the rules of governance for these markets. Following this, our novel technique based on the OPF formulation is applied, which relaxes the inherit strictness of the non-technical PoAs e.g. strict LIFO scheme, while at the same time to control the change in the curtailment levels caused by autonomous $\mathrm{P} 2 \mathrm{P}$ trading. In particular, the changes caused by $\mathrm{P} 2 \mathrm{P}$ that can have negative effect on system operation and overall DG curtailment.

The paper progresses as follows. Section II describes the methodology for modelling the extended formulation of the OPF tool. Section III summaries how the bilateral contracts are modelled for the P2P trades. Section IV presents the model for the case studies and discussion of the results and lastly, Section $\mathrm{V}$ presents the conclusion.

\section{METHODOLOGY}

Initially, an ANM approach was typically modelled using a multi-step power flow problem. However, with an increase of DG connections, initial LIFO based ANM solutions started to curtail significant amounts of generation. This was not always necessary, however, as some of the curtailments did not contribute towards addressing of the network issues. This led to dividing networks into subzones and introducing multiple, that is zonal, LIFO stacks [23]. The problem of the unnecessary DG curtailment lead to the development of tools that were based on Optimal Power Flow (OPF) approach [20]. It is worth noting that, in addition to [20], OPF formulation can also be developed to follow the precise LIFO order. This paper presents such a formulation, which enables an investigation into operation with both strict/current LIFO principles and more flexible Technical Best LIFO (TB-LIFO), as presented below. In addition, these new OPF extended models allow comparisons of operations with and without $\mathrm{P} 2 \mathrm{P}$ trading.

Typically, OPF models are formulated as a nonlinear optimisation problem that aims to minimise system operation costs, subject to network constraints and nodal power balance equations. In this paper, the tool developed to evaluate P2P trading in networks with both strict and Technical Best LIFO ANM, is expressed as a nonlinear optimisation problem. In such a case, the standard OPF formulation is extended to include a set of bilateral contracts and generation cost functions that are adjusted to enable the modelling of different LIFO approaches. This section describes a general methodology to extend the AC OPF model to include each of the LIFO approaches, as well as particular modelling requirements for each of these approaches.

\section{A. Extended AC OPF Model to include LIFO Approaches}

The objective function for the extend ACOPF is a minimisation of the total generation cost:

$$
f(x)=\min \sum_{i=1}^{i} C_{i}\left(P_{g i}\right)
$$

where $C_{i}$ is the cost function for the active power output of generator $i$. For the full AC OPF problem, this objective function is subject to three prominent set of constraints: equality and inequality constraints $g(x)$ and $h(x)$, as well as maximum and minimum limits of the state and control variables, $\mathbf{x}$.

$$
\begin{gathered}
g(x)=0 \\
h(x) \leq 0 \\
\mathbf{x}_{\min } \leq \mathbf{x} \leq \mathbf{x}_{\max }
\end{gathered}
$$

In the standard OPF optimisation vector $\mathbf{x}$ is defined as:

$$
\mathbf{x}=\left[\begin{array}{llll}
\mathbf{P}_{\mathbf{g}} & \mathbf{Q}_{\mathbf{g}} & \mathbf{V}_{\mathbf{m}} & \mathbf{V}_{\delta}
\end{array}\right]^{T}
$$

where $\mathbf{P}_{\mathrm{g}}$ and $\mathbf{Q}_{\mathrm{g}}$ are vectors of generator real and reactive power injections, while $\mathbf{V}_{\boldsymbol{\delta}}$ and $\mathbf{V}_{\mathbf{m}}$ are vectors of voltage angles and magnitudes, respectively. Equations (2) represent 
the mathematical formulation of the static OPF equations, i.e. the power balance equations for both the real and reactive components, expressed as functions of $\mathbf{V}_{\boldsymbol{\delta}}, \mathbf{V}_{\mathbf{m}}, \mathbf{P}_{\mathbf{g}}$ and $\mathbf{Q}_{\mathbf{g}}$, under the assumption that the load injections are constant:

$$
\begin{gathered}
P_{g i}-P_{d i}=P_{i}\left(\mathbf{V}_{m}, \mathbf{V}_{\delta}\right)=\sum_{\substack{j=1 \\
j \neq i}}^{N_{\text {lines }}} P_{i j}\left(\mathbf{V}_{m}, \mathbf{V}_{\delta}\right) \\
Q_{g i}-Q_{d i}=Q_{i}\left(V_{m}, V_{\delta}\right)=\sum_{\substack{j=1 \\
N_{\text {lines }}}} Q_{i j}\left(V_{m}, V_{\delta}\right)
\end{gathered}
$$

In (6) and (7) subscript $d$ denotes the real and reactive demand. Inequality constraints (3) include the minimum and maximum operating points of the optimisation vector $\mathbf{x}$. i.e.:

$$
\begin{aligned}
\mathbf{V}_{m, \text { min }} & \leq \mathbf{V}_{m} \leq \mathbf{V}_{m, \text { max }} \\
\mathbf{P}_{g, \text { min }} & \leq \mathbf{P}_{g} \leq \mathbf{P}_{p, \text { max }} \\
\mathbf{Q}_{g, \text { min }} & \leq \mathbf{Q}_{g} \leq \mathbf{Q}_{p, \text { max }}
\end{aligned}
$$

In addition to constraints (8)-(10), objective (1) is also subject to thermal line limits:

$$
\left|\mathbf{S}_{\text {flow }}\right| \leq \mathbf{S}_{\text {flow, max }}
$$

where $\mathbf{S}_{\text {flow, max }}$ is the apparent power thermal limit vector.

In this paper, the objective function for the OPF (1), is adjusted to distinguish models used for each of the LIFO approaches, i.e., strict/current and Technical Best, using Pseudo cost functions. The constraints (6) - (11), as well as decision variables (5) are the same for both formulations

It is important to note that the cost functions used in both cases do not directly reflect the actual cost of generation, but rather their place in a LIFO priority list. As a result, OPF solution will determine the generation levels to meet demand, which will also reflect that of LIFO principles.

However, under certain conditions, such as voltage congestion, there could be two approaches to address these issues. One, most widely applied in the UK, is to strictly curtail outputs of all generators that has lower priorities in LIFO stack, until the problem is resolved. The other, which is similar to approaches which use not only main, but also zonal LIFO stacks, seeks to find a solution that will follow LIFO stack priories, but will curtail only output of generators that can resolve the problem, while those that have no affect will remain curtailed. In other words, LIFO principles are bypassed and rather than import from the Grid Supply Point (GSP), the OPF dispatch another DG since all DGs would be cheaper than the GSP.

In this paper, the first approach is referred to as Strict $L I F O$, while latter is referred to as Technical Best ANM approach, which is a more flexible form of LIFO arrangement, and allows changes to the outputs of the DGs that would be curtailed otherwise. It is noted that the OPF is solved in a centralised manner, which draws attention to concerns surrounding privacy. Although addressing these concerns is out with the scope of this paper, it is noted that there are developments in smart grid architecture that allows for secure and autonomous transactions, in a manner that is scalable and computationally light [22].

\section{B. Strict LIFO OPF with Pseudo Cost Curves}

To model strict LIFO principles, a multivariate piecewise function was developed so that when there is a constraint, the DG cost curves are adjusted in a manner that will make the GSP the significantly 'cheaper' option. Here, the piecewise function is defined by multiple sub-functions, where each applies to a certain interval of the main function's domain. These intervals are defined by outputs of higher priority generators.

The multivariate surface (in Fig 1) illustrates how the cost of generating from lower priority $\mathrm{DG}_{\mathrm{p}-1}$ is dependent on the output of higher priority $\mathrm{DG}_{\mathrm{p}}$, where the subscript $p$ notates the priority in the LIFO order. The two prominent surfaces describe two operational situations, that is, $\mathrm{DG}_{\mathrm{p}}$ operating or not operating at its maximum capacity. For example, if $D_{\mathrm{p}}$ is not at capacity, which in the case for Fig 1 is $11 \mathrm{MW}$, then the costs of $D G_{p-1}$ would shift to the LHS surface. This adjustment would then cascade across each DG within the LIFO stack (that are lower in the priority list). Situating the cost of the GSP between these two surfaces makes it 'cheaper' than lower priority DGs (when $\mathrm{DG}_{\mathrm{p}}$ is not at capacity) and is hence used over them and thus curtails DGs in accordance to strict LIFO principles.

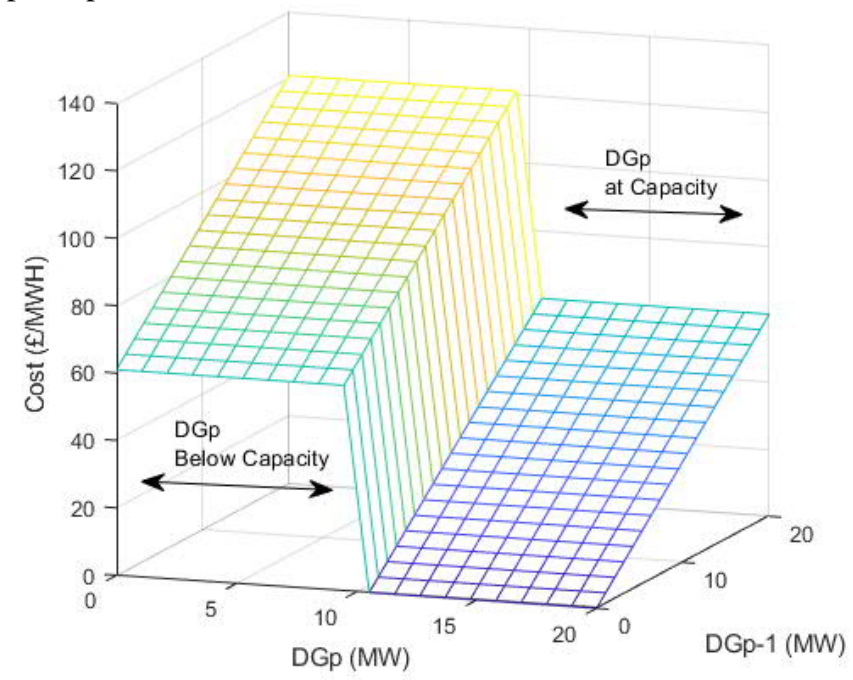

Figure 1. Pseudo LIFO multivariate Cost function

To overcome issues surrounding non-smooth piecewise function (i.e. local minima convergence issues), the cost of the GSP is situated between the highest point of RHS surface and lowest point of the LHS surface (Fig 1), that is, it will always be in the plane that connect these two surfaces but not at the edges of any of these surfaces. Although a continuous surface is used to model DG costs, it essentially consists of a series of 
cross-sections for each DG output interval, which then define a linear polynomial cost function. This takes the form of:

$$
C_{i}\left(P_{g i}\right)=c_{0} P_{g i}
$$

where $\mathrm{c}_{0}$ is the polynomial constant that reflects both the DGs LIFO stack position and the respective surface on the multivariate cost function. The cost function defined in (12) is then used in the objective function of OPF (1).

\section{TB-LIFO ANM Tool}

As mentioned above, the strict LIFO assessment principle of access suffers from multiple drawbacks because real-time curtailment is not based fully on a need to resolve network issues, but rather on the connection order and LIFO list. Moreover, strict LIFO can lead to an underutilised renewable energy as some DGs can be unnecessarily curtailed and replaced by the grid supply.

The overarching goal of this new TB-LIFO tool is to maximise DG capacity on the network whilst ensuring that the determined solution does not cause any unnecessary out of merit curtailment in the LIFO priority order. Therefore, it allows generation of lower priority DGs if, and only if, curtailment of their output levels would not contribute to resolving the network issue.

The TB-LIFO is based on the extended AC OPF formulation (1)-(11), where optimisation aims to maximise DG generation by minimising costs which do not directly reflect the cost of generation, but rather their place in a LIFO priority list. As such, these costs are here referred to as pseudo-costs. Each DG is assigned a pseudo-cost function that takes the form of (12), however now the respective polynomial constant only reflects its position within the stack. Additionally, the GSP is assigned a polynomial constant much higher than that of the LIFO stack.

This TB-OPF ANM tool can be used to analyses levels of unnecessary curtailment compared to the Strict LIFO approach, as well as an influence of levels of P2P trading on DG outputs and overall operation in distribution networks. In addition, twostep optimisation can be developed and used to compare $\mathrm{P} 2 \mathrm{P}$ trading under more strict LIFO arrangements.

\section{P2P TRADING MODELS}

In this paper, the $\mathrm{P} 2 \mathrm{P}$ trading model consists of market environment that allows for peers to directly negotiate and agree upon bilateral contracts, which consist of the magnitude of the power exchange and price. In this market, any generating peer can negotiate and form any bilateral contract with any demand at any bus. In other words, this paper is simulating the environment where a fully $\mathrm{P} 2 \mathrm{P}$ trading mechanism is realised and does not have any conventional intermediaries overseeing the exchanges, meaning the trades are physical. The purpose of doing so is to illustrate the impacts that $\mathrm{P} 2 \mathrm{P}$ trading can have on network operation and DG curtailment, and, in turn, inform the holistic control and congestion management strategies required when considering LIFO DGs and $\mathrm{P} 2 \mathrm{P}$ trading. Furthermore, the $\mathrm{P} 2 \mathrm{P}$ trades are physical with privately negotiated prices and therefore these prices are not considered by the OPF calculation.

\section{A. Bilateral Contract Modelling for P2P Trades}

Let the vector of total demand for all $n$ buses be denoted by $\mathbf{P}_{\mathbf{d}}$, which can then be decomposed into two components: the sum of load provided by entering into privately negotiated $\mathrm{P} 2 \mathrm{P}$ trade, and the remaining part that is bought in an usual manner from the supplier (here designated as a central pool [21].) The total consumption then consists of the two components:

$$
\mathbf{P}_{\mathbf{d}}=\mathbf{P}_{\mathbf{d}}^{p}+\mathbf{P}_{\mathbf{d}}^{c}
$$

where the superscript $p$ and $c$ are the demand met by the P2P bilateral trades and central pool, respectively. Similarly, the total generation vector for all buses, $\mathbf{P}_{\mathbf{g}}$, can also be decomposed into trades through corresponding P2P contracts, and central pool, which in the case of the DG can be an aggregator or similar, i.e.:

$$
\mathbf{P}_{\mathrm{g}}=\mathbf{P}_{\mathrm{g}}^{p}+\mathbf{P}_{\mathrm{g}}^{c}
$$

Assuming that every DG can negotiate a P2P trade with a demand at any bus, those trades can be expressed as a matrix denoted by GD. For example, if DG at bus 2 entered into a P2P contract with a load at bus 5 set at $1 \mathrm{MW}, \mathrm{GD}_{i=2, j=5}$ would therefore be set to $1 \mathrm{MW}$. Consequently, summation of the power scheduled through P2P trades, GD, plus the central pool schedule will equal the total system demand,

$$
\text { Power }_{\text {req }}=\text { GD }+ \text { Pool }
$$

Note that OPF formulation includes provision of losses, which are supplied by the central pool component for all trades. It must be noted that in this paper, the resultant GD matrix will add an additional constraint to the above OPF formulation. This constraint models "firm" nature of the P2P trades, as both parties involved in the trade are obliged to fulfil their obligations. This means that minimum level of DG output cannot be below the total of its $\mathrm{P} 2 \mathrm{P}$ obligations,

$$
\mathbf{P}_{g, i} \geq \mathbf{P}_{g, i}^{P}=\sum_{j=1}^{n_{b u s}} G D_{i, j}
$$

Therefore, the P2P schedule of the DGs in the GD matrix is added as additional new lower bounds for the respective generator unit in the OPF optimisation.

The above modelling of the $\mathrm{P} 2 \mathrm{P}$ trading is implemented for the TB-OPF ANM approach because these trades are arranged outside of the centralised operational mechanisms. It would be possible to model P2P trading within a Strict LIFO approach, although such arrangements will not be as beneficial as in the case of TB-OPF as generators are curtailed regardless of their effect on issues in network operation. Under those conditions, a two-step procedure would be used, where, first, the pseudo LIFO cost curves from Section B would be used to determine the strict LIFO dispatch. This output would then be set as a 
new lower bound, $P_{g i, m i n}$, on DGs outputs, so that for each respective generator constraint defined in (9) becomes:

$$
P_{g i} \geq P_{g i, \min }
$$

Once $P_{g i \text { min }}$ is set, the TB-LIFO would use any spare capacity on the network in a manner that maximizes DG output.

\section{Simulation Results AND Discussion}

In this section, we present three case studies. Firstly, we present the 'Base Case' that illustrates how DG curtailment can change with LIFO ordering under strict LIFO principles. Following this, 'Case Study 2' demonstrates the varied, and in some cases significant, impact that $\mathrm{P} 2 \mathrm{P}$ trading can have on the curtailment of DGs under different LIFO priority ordering. This is carried out under strict LIFO principles. Lastly, in 'Case Study 3', we implement the proposed ANM solution, that uses the TB-OPF tool to optimise the DGs whilst respecting bilateral contracts from the $\mathrm{P} 2 \mathrm{P}$ trading. The simulations and extended AC OPF model have been implemented in conjunction with the MATPOWER suite for power system analysis using Matlab.

\section{A. Test System and Setup}

The network used for the case studies is considered as a representative of a radial low voltage UK distribution network, from United Kingdom Generic Distribution System (UKGDS) [24]. UKGDS is a resource for simulation and analysis of UK power networks when addressing the impacts of DGs. The network chosen, as shown in Figure 2, is an $11 \mathrm{kV}$ rural radial network fed from a $33 \mathrm{kV}$ supply point. There is a total of 76 buses all of which follow the statutory voltage limit of $\pm 6 \%$ of nominal. Three DGs were installed at bus numbers 55, 20 and 42 with capacities of $15,10.5$ and $11.5 \mathrm{MW}$ respectively. Here, the capacities were selected based on the maximum DG capacity of the network at a peak demand, whereby each generator was allowed to export (through the GSP) as much power as the physical network could allow. The exporting of power was encouraged through the polynomial cost curve of the GSP in the OPF solution, where negative power generation (necessary for modelling of exporting of DGs) was actually in net benefit to the total cost minimisation problem.

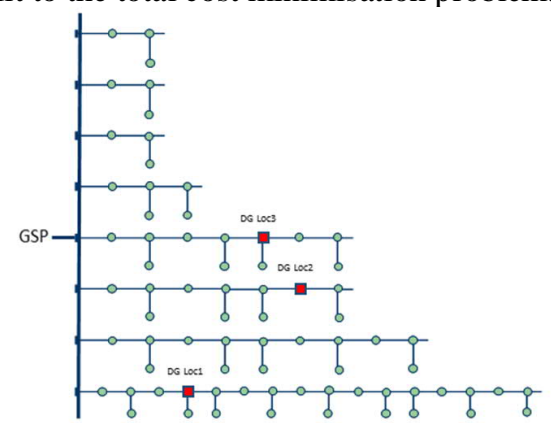

Figure 2. Network Diagram of case study network with 3 DGs.
Under an ANM scheme, these three DGs would have been connected and contracted in accordance to strict LIFO curtailment principles. This creates a possible six unique scenarios of priority orders, depending on which DG was first installed. The priority order of the DGs is provided in Table 1, where ' $L$ ' DGs will be curtailed first while those of the priority order of " $\mathrm{H}$ " last. For all the case studies, each scenario is simulated and compared. Furthermore, each case study is simulating a period of constraint meaning the GSP cannot export power.

Table 1: Description of 6 Scenarios of 3 DGs with varying LIFO priority

\begin{tabular}{c|c|c|c}
\hline \hline \multirow{2}{*}{ Scenario } & \multicolumn{3}{|c}{ Priority(High=H, Medium=M, Low=L) } \\
\cline { 2 - 4 } & DG LOC1 & DG LOC 2 & DG LOC 3 \\
\hline \hline 1 & $\mathrm{H}$ & $\mathrm{M}$ & $\mathrm{L}$ \\
\hline 2 & $\mathrm{H}$ & $\mathrm{L}$ & $\mathrm{M}$ \\
\hline 3 & $\mathrm{~L}$ & $\mathrm{H}$ & $\mathrm{M}$ \\
\hline 4 & $\mathrm{M}$ & $\mathrm{L}$ & $\mathrm{H}$ \\
\hline 5 & $\mathrm{~L}$ & $\mathrm{M}$ & $\mathrm{H}$ \\
\hline 6 & $\mathrm{M}$ & $\mathrm{H}$ & $\mathrm{L}$ \\
\hline \hline
\end{tabular}

\section{B. P2P Matching Process}

Three assumptions are made for the simulations that use P2P trading in this paper. First, for each simulation case studied, only one generator is involved with the P2P bilateral power trades. The generator can create contracts with any and all nodes that have a demand. The second assumption is that demand is considered inelastic, meaning the private contracts drawn cannot be above the total demand at that node, and only influence OPF solution of the DGs outputs. Further work will include the ability for the demand at each node to essentially become prosumers, having abilities to increase or decrease consumption. Lastly, it is assumed that the $\mathrm{P} 2 \mathrm{P}$ matching process is completely random, both in terms of who the generator trades with and the quantity of power agreed. For this paper, it is acknowledged that location of trades may significantly interfere with operation. However, as the demand is inelastic (and will either be supplied by the GSP or DGs) it is only the location of the generation that is of interest.

\section{Base Case: Impacts of LIFO Ordering on DG Capacity}

Curtailment levels can be significant, particularly in voltage constrained areas, and especially when considering the priority ordering of DGs. Here, a Base Case for the 6 strict LIFO scenarios, corresponding to combinations in Table 1, are presented. As stated above, these case studies are simulating an upstream constraint event, meaning GSP cannot export. The total demand of the customers connected at the distribution level is inelastic and equal to $24 \mathrm{MW}$.

From Figure 3, it can be seen that when the generator in ' $D G$ Loc1' has high priority " $\mathrm{H}$ " (Scenarios 1 and 2), total DG output of all generators is able to supply the local demand, 
without requiring supplement from the GSP. Here, any curtailed DG power is actually a consequence of the GSP export constraint rather than local network conditions.

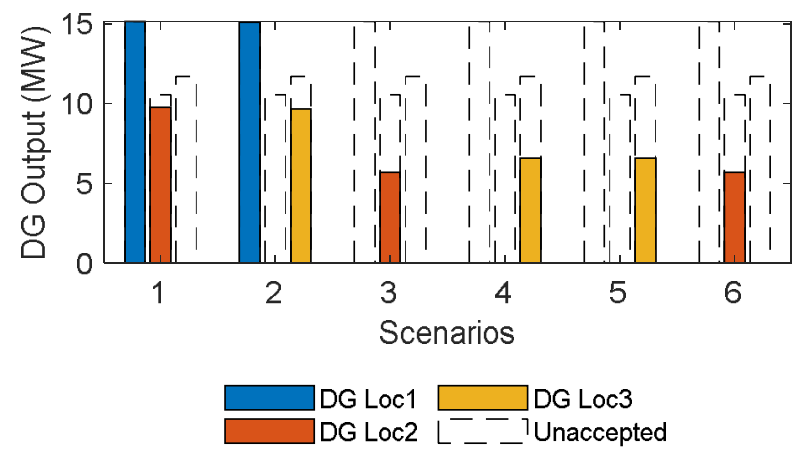

Figure 3: Total DG output across all scenarios for Base Case.

Contrarily, in Scenarios 3 to 6 DG outputs of all generators are either capped to 5.7 or $6.6 \mathrm{MW}$, resulting in the ' $M$ ' and ' $L$ ' priority DGs being curtailed to $0 \mathrm{MW}$. Here, overall curtailment levels are around $79 \%$ or $75 \%$, higher than when compared to the first two scenarios. If we examine the voltage profile of the network where no DGs are installed (the blue trace in Figure 4), it can be seen that the issue on this particular network are low voltages at the end of the longest feeder buses 66 and 76. The addition of a DG provides voltage support, however, the addition of a DG on a parallel feeder, as seen in Scenarios 3 to 6, only amplifies this voltage issues (i.e. when DG at ' $D G$ loc2' or ' $D G$ loc3' is given priority). During these latter Scenarios, voltages on the end of feeder (66 and 76) are forced even lower due to the increase distance from the source. Consequently, to avoid this lower limit constraint, the voltages at the DGs connection points are forced to their upper limits. This causes a cap on the output from these DGs, cascading curtailment of lesser priority generators and therefore relying on imports from the GSP.

In scenarios 1 and 2, greater levels of generation are seen to a point where curtailment is not enforced due to a network but rather export constraints, i.e. local demand equals local generation. In these scenarios, priority is given to generator one, located on the weakest feeder in terms of voltage. The inherit voltage support from the DG in 'DG Locl', not only enables its own maximum output but also releases capacity on the parallel feeders. The now raised voltage on the end of the longest radial feeders allows additional capacity without raising voltages of the respective location on parallel feeders. The capacity released on these previously constrained feeders are around $42 \%$ and $32 \%$ in scenarios $3 / 5$ and $4 / 6$ respectively.

\section{Case Study 2: Impacts of P2P trading on LIFO DGs}

With the expected rise of $\mathrm{P} 2 \mathrm{P}$ trading the concerns of strict LIFO may be amplified, although there are instances when the P2P trading may actually alleviate voltage congestion. Therefore, the analysis of the six scenarios is now extended to include $\mathrm{P} 2 \mathrm{P}$ trading to assess the impact such trading has on strict LIFO DGs. For these simulations P2P trading will be autonomous, firm and cannot be curtailed, which is enforced through (17). This followed the approach detailed in [11] and similar to pilot projects in [6], where a 'Hybrid P2P Market' is established on the network in tandem with external markets (in this case the central pool).

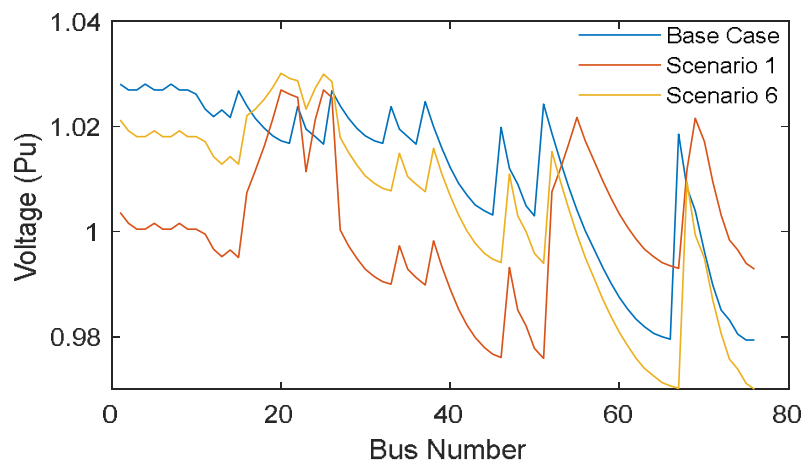

Figure 4: Comparison of voltage profiles

In each of the six scenarios, the last priority generator is given the role of $\mathrm{P} 2 \mathrm{P}$ trading. In reality, it can be expected that a number of generators will seek to engage in P2P trading, however, analysis of those complexities is outside of the scope of this paper. Using a single DG that trades with various participating demand nodes across the network, provides a clearer example of how P2P can influence network operation and more precisely the impacts such trading can have on the LIFO stack.

A series of simulations for each Scenario were run so that the participation level of $\mathrm{P} 2 \mathrm{P}$ trading was proportionally increased. For example, in Scenario 1 ' $D G$ loc3' enters into $\mathrm{P} 2 \mathrm{P}$ contracts, initially set to $10 \%$ participation levels, resulting in the sum of elements of the corresponding row of GD matrix in (13), and similarly in $\mathbf{P}_{\text {gi min }}$ in (14), to be 2.4 MW. This is then increased to $20 \%$, and so on by $10 \%$, until the value of total P2P trades, defined in GD, reaches the capacity of ' $D G$ loc3' which is 11.5 MW. Figure 5 demonstrates the resultant portion of $\mathrm{P} 2 \mathrm{P}$ trades compared to the total scheduled power.

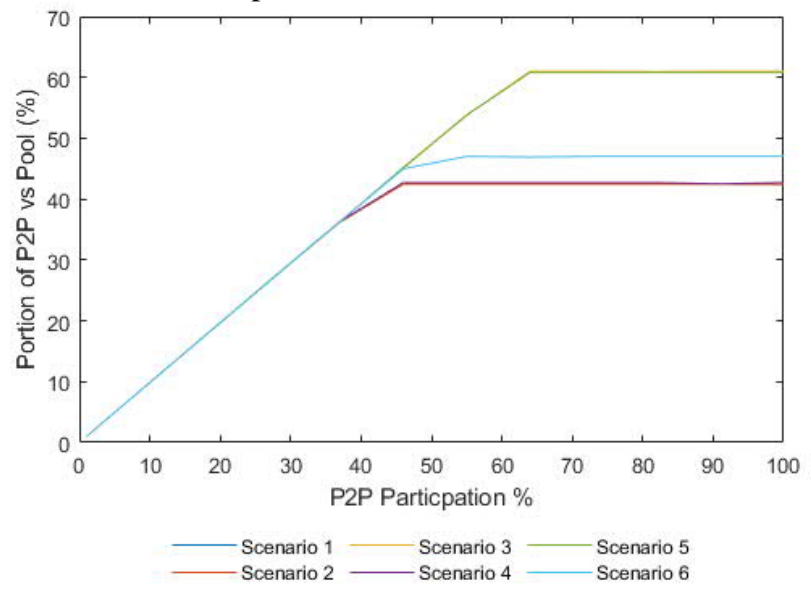

Figure 5: Portion of P2P trades compared to the total scheduled power 
From Figure 6, it can be seen that the results can be broadly split into two outcomes. Scenarios 3, 4 and 5 actually see an improvement in total curtailed DG output, as seen by the 3 negative traces in Figure 5. Here, a negative trace demonstrates a reduction in the level of curtailment from the levels shown in Figure 3. For each of these three scenarios, the total curtailed power incrementally reduces, and then begins to either recede back or plateau. The reduction is seen due to the increase voltage support from the parallel $\mathrm{P} 2 \mathrm{P}$ trading generator, similarly as discussed in Scenario 1 and 2 in the Base case. In an ANM with strict LIFO, the P2P generator would in these cases be forced to $0 \mathrm{MW}$. However, as the $\mathrm{P} 2 \mathrm{P}$ trades are uncurtailable, its output increases the overall DG generation on the network.

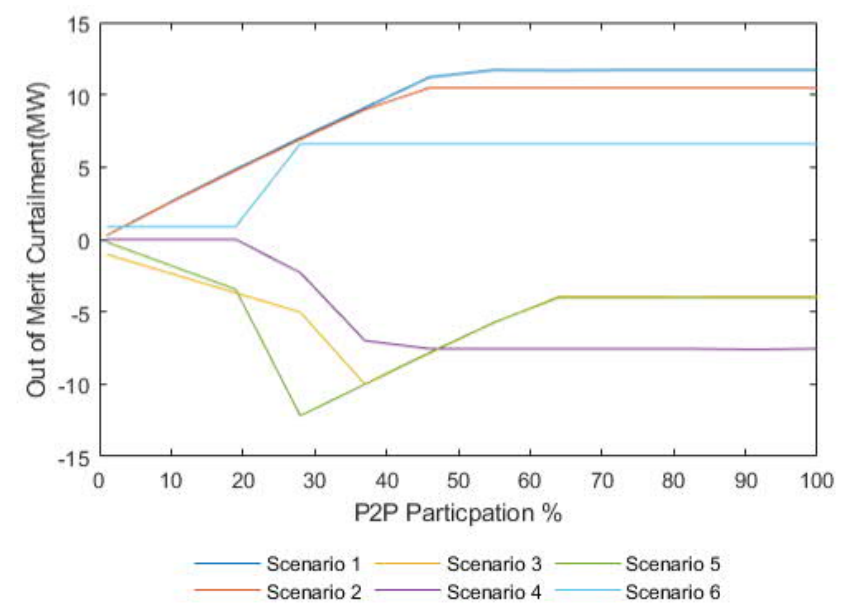

Figure 6: Impacts on curtailment levels from increasing P2P participation

Looking at Scenario 5, where 'DG Locl' is the DG entering in $\mathrm{P} 2 \mathrm{P}$ contracts, we see that around $30 \%$ participation the curtailment actually begins to recede and then plateaus at around $65 \%$. What happens here is that the maximum amount of DG capacity is released, equalling a total of $24 \mathrm{MW}$ and as the P2P increase its output, and all it does is curtail a higher priority DG, although total DG output on the system remains $24 \mathrm{MW}$. In other words, the P2P generator 'skips' the priority order. This can also be said for Scenarios 1 and 2.

For the Scenario 6, however, when $\mathrm{P} 2 \mathrm{P}$ participation levels reached around $30 \%$ infeasible solutions occurred, which in Fig 5 is represented as all DGs being set to $0 \mathrm{MW}$. Here ' $D G$ Loc3' is the P2P trading DG, and as seen from the base case, when this DG exceeds 5.7 MW (without the support from ' $D G$ Locl') it reaches its upper voltage limit. As (14) is here set beyond 5.7 MW it causes non-convergence in the optimisation due to the overloaded bus voltages.

\section{E. Case Study 3: Implementation of TB-OPF ANM Tool}

In this section, the effects introduced by $\mathrm{P} 2 \mathrm{P}$ trading will be managed through our proposed TB-OPF tool. Following the same process as in the latter section, $\mathrm{P} 2 \mathrm{P}$ participation levels were incrementally increased for each of the LIFO ordering scenarios.
The results in Figure 7 show the total curtailed DG output when using the TB-OPF tool compared to the base case shown in Figure 3. It shows that for almost every case (excluding Scenarios 1 and 2), a significant increase in DG output is seen at all levels of $\mathrm{P} 2 \mathrm{P}$ participation. What this demonstrates is that, up to a point, $\mathrm{P} 2 \mathrm{P}$ trading can be managed in a manner that does not see any out of merit curtailment (when compared to DGs operating under strict LIFO principles) and actually, even at $100 \%$ P2P participation levels, the TB-OPF can actively manage the network and allow for these power exchanges. Whereas previously, for Scenario 6, even at $30 \%$ levels of participation there were significant overloads and non-convergence issues.

However, for Scenario 1 and 2 where the traces mirror the respective traces shown in Figure 6, it shows that even with the TB-OPF tool there is no improvement. This is because, for these Scenarios, and as shown in Figure 3, the total DG output without $\mathrm{P} 2 \mathrm{P}$ trading equalled that of demand. Therefore, an increase in the output of the power supplied by the P2P market will always cause a reduction within the LIFO stack.

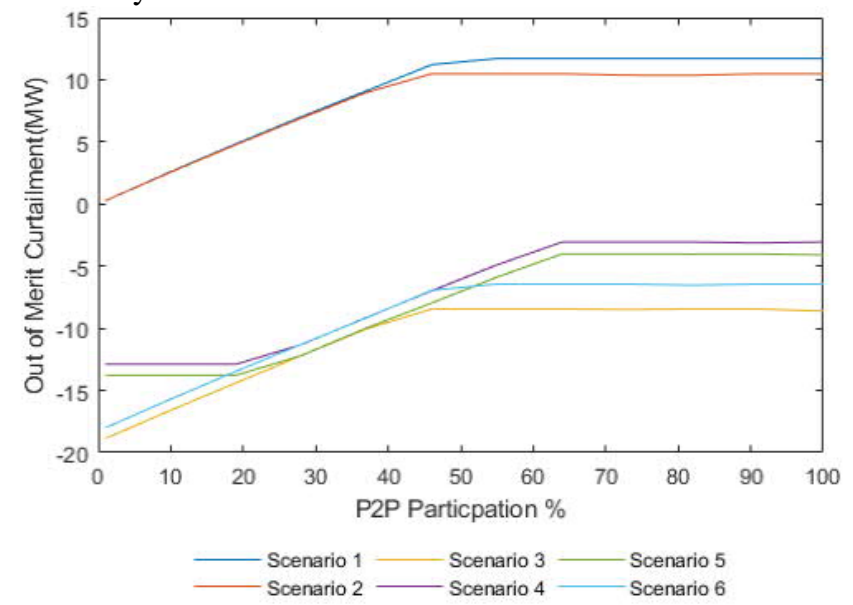

Figure 7: Impacts on curtailment levels using TB-OPF Tool

Figure 8 shows specific output for each DG at $80 \%$ P2P participation.

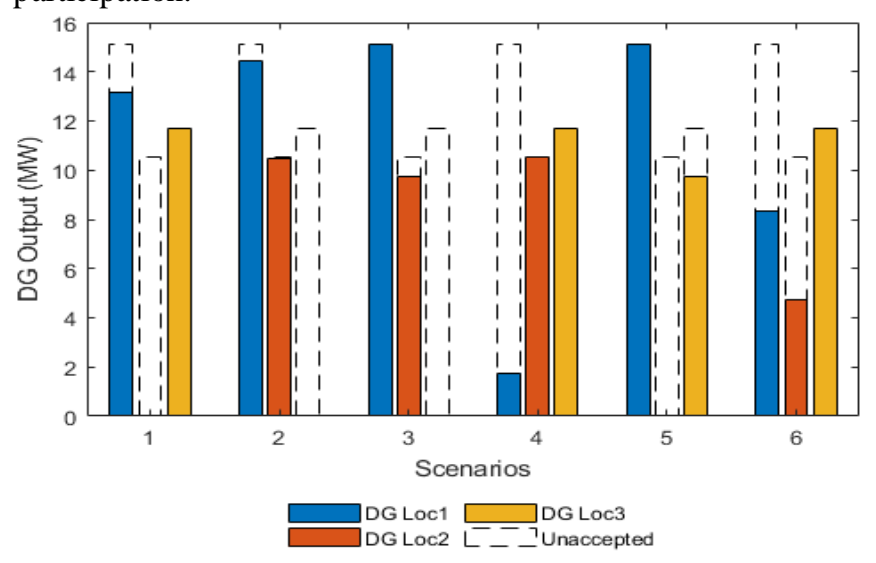

Figure 8: Total DG output across all scenarios for TB-OPF Case. 


\section{CONCLUSION}

This paper presents novel OPF based tools developed to evaluate $\mathrm{P} 2 \mathrm{P}$ trading in networks with both strict and Technical Best LIFO ANM. It is based on a full AC network modelling which can be used for analysis of distribution level networks with high levels of DER connections. The standard OPF formulation is extended to include a set of physical P2P bilateral contracts and generation cost functions that are adjusted to enable the modelling of different LIFO approaches and $\mathrm{P} 2 \mathrm{P}$ trading.

Through a series of case studies, it is investigated how enabling $\mathrm{P} 2 \mathrm{P}$ energy trading without conventional intermediaries impacts network operation. In particular, by allowing the least priority generator to enter into privately negotiated physical P2P bilateral contracts it was possible to analyses how increased levels of the P2P trading under different LIFO approaches can have a significant impact on other DGs in the network, and in some cases can be unavoidable. This confirms a need to evaluate opening of local electricity markets to such trades, as well as a need to better understand, manage and regulate them. It is recommended that the impacts of P2P be comprehensively analysed, with such tools as the AC OPF, before allowing and finalising the rules for this type of trading.

As the paper and the proposed methods only apply to DGs interfaced through the specific PoA LIFO, a typical UK model, the future extension will require modifications to account for different approaches. These approaches can also include different local market arrangements, for example, a market where P2P trading provides ancillary services. Future work will also include investigating these different approaches as well as, investigation of appropriate market signals and/or incentives that avoid inefficient P2P trades.

Although this paper highlights the importance of the full AC OPF, it may be computationally expensive. Therefore, future work will also seek include implementing methods that linearize the optimisation but retain its accuracy such as second order cone programming relaxations.

\section{ACKNOWLEDGEMENTS}

The development of this research is supported by UK Engineering and Physical Sciences Research Council (EPSRC) through Centre for Doctoral Training in Future Power Networks and Smart Grids [EP/L015471/1]. All results can be fully reproduced using the methods and data described in this paper and provided references.

\section{REFERENCES}

[1] C. Bussar et al., "Large-scale integration of renewable energies and impact on storage demand in a European renewable power system of 2050," Energy Procedia, vol. 73, pp. 145-153, 2015.

[2] V. S. Bhadoria, "A Review on Distributed Generation Definitions and DG Impacts on Distribution System," 2013. [Online]. Available https://www.researchgate.net/publication/260095963_A_Review_on_ Distributed_Generation_Definitions_and_DG_Impacts_on_Distributio n_System

[3] N. Grid, "System Operability Framework 2016 ", 2016. [Online] Available:http://www2.nationalgrid.com/UK/Industryinformation/Future-of-Energy/System-Operability-Framework/
[4] ENA, "Active Network Management, Good Practise Guide," 2015. [Online].Available: http://www.energynetworks.org/assets/files/news/ publications/1500205_ENA_ANM_report_AW_online.pdf

[5] R. Currie, B. Oneill, and C. Foote, "COMMERCIAL ARRANGEMENTS TO FACILITATE ACTIVE NETWORK MANAGEMENT," 2011. [Online]. Available: http://www.cired.net/publications/cired2011/part1/papers/CIRED2011 _1186_final.pdf

[6] Chenghua Zhang, Jianzhong Wu, Chao Long, Meng Cheng, "Review of Existing Peer-to-Peer Energy Trading Projects", Energy Procedia, Volume 105, 2017, Pages 2563-2568,ISSN 1876-6102

[7] J. Rodríguez-Molina, M. Martínez-Núñez, J.-F. Martínez, and W. Pérez-Aguiar, "Business models in the smart grid: Challenges, opportunities and proposals for prosumer profitability," Energies, vol. 7, no. 9, pp. 6142-6171, 2014.

[8] F. Hvelplund, "Renewable energy and the need for local energy markets," Energy, vol. 31, no. 13, pp. 2293-2302, 2006.

[9] C. Rosen and R. Madlener, "Regulatory options for local reserve energy markets: Implications for prosumers, utilities, and other stakeholders," FCN Working Papers 12/2014, E.ON Energy Research Center, Future Energy Consumer Needs and Behavior (FCN).

[10] T. Linnenberg, I. Wior, S. Schreiber, and A. Fay, "A market-based multi-agent-system for decentralized power and grid control," in ETFA2011, 2011: IEEE, pp. 1-8.

[11] Y. Parag and B. K. Sovacool, "Electricity market design for the prosumer era," Nature energy, vol. 1, no. 4, p. 16032, 2016.

[12] E. Sorin, L. Bobo, and P. Pinson, "Consensus-based approach to peerto-peer electricity markets with product differentiation," IEEE Transactions on Power Systems, 2018.

[13] T. Morstyn, A. Teytelboym, and M. D. McCulloch, "Bilateral contract networks for peer-to-peer energy trading," IEEE Transactions on Smart Grid, 2018.

[14] N. Liu, X. Yu, C. Wang, C. Li, L. Ma, and J. Lei, "Energy-sharing model with price-based demand response for microgrids of peer-to-peer prosumers," IEEE Transactions on Power Systems, vol. 32, no. 5, pp. 3569-3583, 2017.

[15] D. Ilic, P. G. Da Silva, S. Karnouskos, and M. Griesemer, "An energy market for trading electricity in smart grid neighbourhoods," in 2012 6th IEEE International Conference on Digital Ecosystems and Technologies (DEST), 2012: IEEE, pp. 1-6.

[16] C. Long, J. Wu, C. Zhang, M. Cheng, and A. Al-Wakeel, "Feasibility of peer-to-peer energy trading in low voltage electrical distribution networks," Energy Procedia, vol. 105, pp. 2227-2232, 2017.

[17] T. Liu, X. Tan, B. Sun, Y. Wu, X. Guan, and D. H. Tsang, "Energy management of cooperative microgrids with $\mathrm{p} 2 \mathrm{p}$ energy sharing in distribution networks," in 2015 IEEE international conference on smart grid communications (SmartGridComm), 2015: IEEE, pp. 410-415.

[18] T. Baroche, P. Pinson, R. L. G. Latimier and H. B. Ahmed, "Exogenous Cost Allocation in Peer-to-Peer Electricity Markets," in IEEE Transactions on Power Systems, vol. 34, no. 4, pp. 2553-2564, July 2019.

[19] J. Guerrero, A. C. Chapman, and G. Verbič, "Decentralized P2P energy trading under network constraints in a low-voltage network," IEEE Transactions on Smart Grid, 2018.

[20] Dolan, M. J., Davidson, E., Kockar, I., Ault, G., \& McArthur, S. (2012). Distribution power flow management utilising an online optimal power flow technique. IEEE Transactions on Power Systems, 27(2), 790-799.

[21] Cuervo Franco, P., Galiana, F., \& Kockar, I. (2002). Combined pool/bilateral dispatch part1: performance of mixed trading strategies. IEEE Transactions on Power Systems, 17(1), 92-99.

[22] N. Z. Aitzhan and D. Svetinovic, "Security and Privacy in Decentralized Energy Trading Through Multi-Signatures, Blockchain and Anonymous Messaging Streams," in IEEE Transactions on Dependable and Secure Computing, vol. 15, no. 5, pp. 840-852, 1 Sept.Oct. 2018.

[23] Access Details Here: https://www.ssen.co.uk/ANM/

[24] UKGDS, "UK Generic Distribution System," 2015. [Online]. Available: https://github.com/sedg/ukgds 